\title{
DISTRIBUIÇÃO E PROBABILIDADE DE OCORRÊNCIA DE PRECIPITAÇÃO EM CÁCERES (MT) ${ }^{1}$
}

\author{
Jaqueline Aguilla Pizzato ${ }^{2}$, Rivanildo Dallacort², Rafael César Tieppo ${ }^{2}$, \\ Alcir José Modolo ${ }^{3}$, Cassiano Cremon², Patrícia Simone Palhana Moreira ${ }^{2}$
}

\begin{abstract}
DISTRIBUTION AND PROBABILITY OF RAINFALL OCCURRENCE IN CÁCERES, MATO GROSSO STATE, BRAZIL
\end{abstract}

Rainfall is an important meteorological element, and the knowledge on its historical records is relevant for monitoring the impacts caused by its prolonged excess or lack. This study aimed to evaluate the monthly and annual rainfall variability and the probability for its occurrence, by using the incomplete Gamma distribution model, for the rainfall data from Cáceres, Mato Grosso State, Brazil. A series of 26 years data, provided by the National Institute of Meteorology, which supports a station in Cáceres, was used. The annual rainfall amounts showed great variability, with minimum of $972.9 \mathrm{~mm}$, in 1985, and maximum of $1,624.1 \mathrm{~mm}$, in 1998 . The monthly data pointed out two well defined seasons: the dry season (May to September) and the rainy one (October to April). The lowest monthly averages occurred in June, July, and August, with $16.59 \mathrm{~mm}, 17.90 \mathrm{~mm}$, and $20.09 \mathrm{~mm}$, respectively. The estimates for the $\alpha$ parameter ranged from 0.9, in June and August, to 13.4, in March. The $\beta$ parameter ranged from 13.2, in March, to 33.1, in January. The highest rainfall probability takes place from December to March, while June, July, and August show the lowest rainfall probability.

KEY-WORDS: Incomplete Gamma function; climate; meteorology.

\section{INTRODUÇÃO}

O Pantanal possui a maior planície inundável da América do Sul, com cerca de $140.000 \mathrm{~km}^{2}$, ocupando 1,6\% do território brasileiro. Situado entre a Amazônia, o Cerrado e os Chacos Boliviano e Paraguaio, é considerado um conjunto de vários ecossistemas, provenientes dos rios da Bacia do Alto Paraguai (Iversson et al. 1993, Cunha \& Junk 2004).

A região do Pantanal, na qual se insere o município de Cáceres (MT), apresenta clima tropical,

\section{RESUMO}

A precipitação é um elemento meteorológico de grande importância, e seu conhecimento histórico torna-se relevante para o monitoramento de impactos causados pelo seu excesso ou falta prolongados. Objetivou-se, no presente trabalho, analisar a distribuição da precipitação mensal e anual e os níveis de probabilidade de ocorrência de chuvas, utilizando-se o modelo probabilístico de distribuição Gama incompleta, para os dados de precipitação pluviométrica de Cáceres (MT). Utilizou-se uma série de dados de 26 anos, disponibilizada pelo Instituto Nacional de Meteorologia, o qual possui uma estação em Cáceres. A precipitação anual teve grande variabilidade, com mínima de 972,9 mm, em 1985, e máxima de 1.624,1 mm, em 1998. Observou-se, por meio dos dados mensais, que existem duas estações bem definidas, a seca (maio a setembro) e a chuvosa (outubro a abril). As menores médias mensais ocorreram nos meses de junho, julho e agosto, com 16,59 mm, $17,90 \mathrm{~mm}$ e $20,09 \mathrm{~mm}$, respectivamente. As estimativas do parâmetro $\alpha$ variaram de 0,9 , em junho e agosto, a 13,4, em março. O parâmetro $\beta$ variou de 13,2 , em março, a 33,1, em janeiro. $\mathrm{O}$ período com maior probabilidade de precipitação vai de dezembro a março, enquanto junho, julho e agosto foram os meses em que a probabilidade de ocorrência de precipitação foi mais baixa.

PALAVRAS-CHAVE: Função Gama incompleta; clima; meteorologia.

com temperatura do ar elevada o ano todo, e médias anuais que podem variar de $22,4^{\circ} \mathrm{C}$, no mês de julho, a $27,3^{\circ} \mathrm{C}$, no mês de outubro (Dallacort et al. 2010).

A precipitação é um elemento meteorológico de grande importância, que pode influenciar, direta ou indiretamente, diversos setores da economia, como, por exemplo, o agropecuário (Silva et al. 2007). Segundo Salgueiro (2005), o conhecimento dos totais precipitados constitui elemento importante na determinação das vazões superficiais e, consequentemente, de suas disponibilidades,

1. Trabalho recebido em maio/2011 e aceito para publicação em fev./2012 (n registro: PAT 14144).

2. Universidade do Estado de Mato Grosso, Departamento de Agronomia, Tangará da Serra, MT, Brasil.

E-mails: japizatto@gmail.com, rivanildo@unemat.br, rafaeltieppo@yahoo.com.br, cassiano.cremon@unemat.br, patriciapalhana@gmail.com.

3. Universidade Federal Tecnológica do Paraná, Campus de Pato Branco, Pato Branco, PR, Brasil. E-mail: alcir@utfpr.edu.br. 
para um gerenciamento sustentável dos recursos hídricos.

Para Vela et al. (2007), o conhecimento do histórico da precipitação é de suma importância para o monitoramento dos impactos causados pelo excesso ou falta prolongada de precipitação, em determinada região. Na região do Pantanal, o conhecimento sobre o regime pluviométrico pode influenciar desde a atividade agropecuária até as previsões de alagamentos, em regiões de riscos.

As informações sobre o regime pluviométrico no Pantanal são de fundamental importância, também, para a produção de gado de corte, principal atividade econômica da região, pois sua quantidade e distribuição, durante o ano, têm influência sobre os regimes de cheia e seca, os quais condicionam uma maior ou menor disponibilidade de pastagens para o gado. Em anos de cheia, as áreas mais baixas, que possuem pastagens de melhor qualidade, ficam cobertas por água, durante períodos que dependem da intensidade e duração da precipitação, prejudicando a pecuária (Soriano \& Galdino 2005).

Há inúmeros benefícios proporcionados pelo Pantanal Matogrossense ao Estado, mas é, também, evidente a preocupação com a sua vulnerabilidade hidrológica, que afeta não somente a fauna e a flora, como, também, as mudanças climáticas, que podem alterar as vazões dos rios, bem como os elementos que dão sustentabilidade a este bioma, influindo, diretamente, na sociedade e na economia das cidades que dele dependem (Diniz \& Fonseca 2008).

O município de Cáceres é banhado pelo Rio Paraguai e destaca-se como pólo turístico do Pantanal Matogrossense e, ainda, por possuir um dos maiores rebanhos bovinos da Região Centro-Oeste do Brasil. Tais características tornam a economia desta região dependente do regime pluviométrico (Tenuta 2004). Por esta razão, este trabalho objetivou analisar a distribuição da precipitação mensal e anual e os níveis de probabilidade de ocorrência de chuvas, no município de Cáceres (MT).

\section{MATERIAL E MÉTODOS}

Os dados pluviométricos foram disponibilizados pelo Instituto Nacional de Meteorologia, o qual possui uma estação pluviométrica localizada no município de Cáceres $\left(16^{\circ} 03^{\prime} \mathrm{S}, 57^{\circ} 41^{\prime} \mathrm{W}\right.$ e altitude de $118 \mathrm{~m}$ ). Os valores de precipitação foram coletados com o auxílio de um pluviômetro tipo Ville de Paris, instalado sobre suporte de $1,5 \mathrm{~m}$ de altura do solo, de 1979 a 2009 (30 anos). No entanto, não foram considerados os anos de 1991, 1993, 1994, 1996 e 1997, devido a falhas nos equipamentos, durante a coleta de dados. Desta forma, foram analisados dados de 26 anos.

Os dados foram tabulados e sua consistência verificada com o auxílio do software CLIMA, desenvolvido pelo Instituto Agronômico do Paraná (Faria et al. 2003). Posteriormente, foram analisadas as médias e os desvios-padrão mensais e anuais.

A determinação de diferentes níveis de probabilidade de precipitação foi realizada utilizando-se o modelo probabilístico de distribuição Gama incompleta (Thom 1958). Sua função densidade de probabilidade é dada pela seguinte equação:

$$
f(x)=\frac{1}{\beta^{\alpha} \Gamma(\alpha)} x^{\alpha-1} e^{-x / \beta}
$$

em que $\alpha$ é o parâmetro de forma (adimensional), $\beta$ o parâmetro de escala $(\mathrm{mm}), e$ a base do logaritmo neperiano, $x$ o total de precipitação $(\mathrm{mm})$, e $\Gamma$ o símbolo da função Gama, definida conforme a equação que se segue:

$$
\Gamma(\alpha+1)=\sqrt{2 \pi \alpha} \alpha^{\alpha} e^{\alpha}\left(1+\frac{1}{12 \alpha}+\frac{1}{288 \alpha^{2}}-\frac{139}{51840 \alpha^{3}}\right) .
$$

Um dos métodos usualmente utilizados é o método dos momentos, que consiste em igualar a média $(\bar{X})$ e a variância $\left(\mathrm{S}^{2}\right)$ da amostra à média $\mathrm{e}$ à variância da população, originando as seguintes equações (Assis et al. 1996):

$$
\alpha=\frac{\bar{X}^{2}}{S^{2}} \quad \text { e } \beta=\frac{S^{2}}{\bar{X}},
$$

em que $\bar{X}=$ precipitação média do período $(\mathrm{mm})$ e $\mathrm{S}^{2}=$ variância $\left(\mathrm{mm}^{2}\right)$.

Para a análise de ocorrência de dias secos e chuvosos, foram considerados secos os dias em que a precipitação foi igual ou menor a $5,0 \mathrm{~mm}$, e chuvosos quando superior a 5,0 mm (Sans et al. 2001).

\section{RESULTADOS E DISCUSSÃO}

Houve grande variabilidade nos níveis de precipitação, durante os anos avaliados, com mínima de 
972,9 mm, em 1985, e máxima de 1.624,1 mm, em 1998. A média observada, durante os 26 anos, foi de 1.268,4 mm, com desvio-padrão de 148,1 mm (Figura 1). Para Diamantino (MT), região na qual está localizada uma das nascentes do Rio Paraguai, o qual banha todo o Pantanal, Dallacort et al. (2010) constataram média anual de precipitação de 1.815,5 mm, valor superior ao observado para Cáceres.

Conforme Rosa et al. (2007), em estudos sobre a distribuição parcial da precipitação na porção centro-oeste do Estado de Mato Grosso, utilizando-se um período de 10 anos de dados, foram encontradas variações pluviométricas que oscilaram entre $1.700 \mathrm{~mm}$ e $2.000 \mathrm{~mm}$, nos municípios de Brasnorte, Campos de Júlio, Campo Novo do Parecis, Comodoro, Juína, Nova Lacerda, Nova Marilândia, Sapezal e Tangará da Serra, localizados nas porções oeste, sul e norte do Estado de Mato Grosso. Estes valores são superiores aos encontrados no presente estudo, que apresentou média anual de $1.268,4 \mathrm{~mm}$ de precipitação, para o município de Cáceres.

Ao estudar a bacia hidrográfica Paraguai/Jauquara, localizada no Estado de Mato Grosso, Casarin et al. (2008) relataram que, para a região de Cáceres, a precipitação média anual é de $1.280 \mathrm{~mm}$.

De acordo com a análise dos dados da estação pluviométrica de Cáceres, observa-se que, em $42 \%$ dos anos (11 anos), os totais de precipitação foram superiores à média, e, em 58\% dos anos (15 anos), abaixo da média (Figura 1).

As médias mensais dos 26 anos avaliados caracterizam duas estações bem definidas na região: a seca (maio a setembro) e a chuvosa (outubro a abril) (Figura 2). Casarin et al. (2008) encontraram a mesma

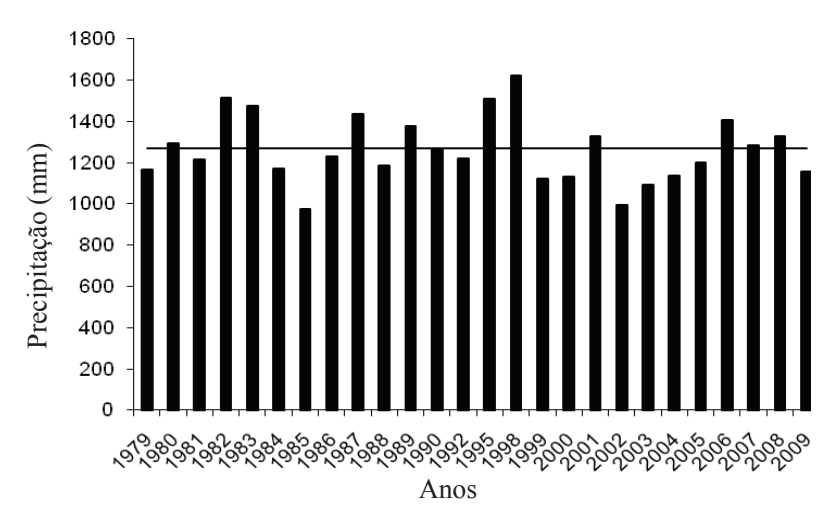

Figura 1. Distribuição da precipitação anual, para o município de Cáceres (MT), de 1979 a 2009. A linha horizontal representa a média da precipitação.

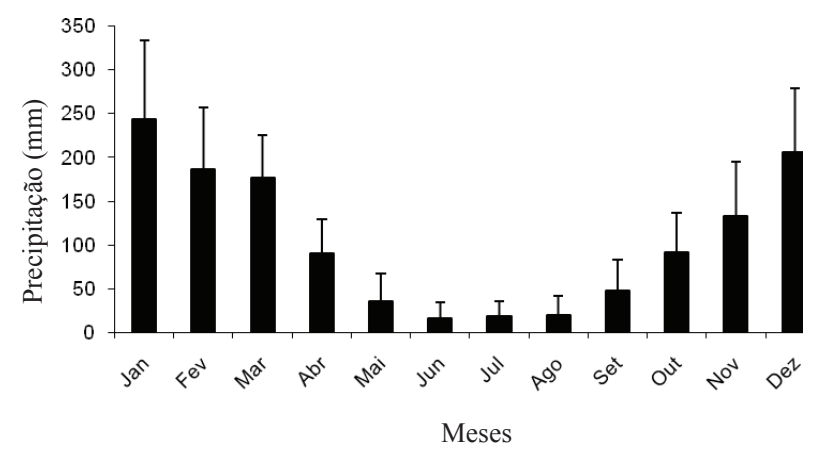

Figura 2. Distribuição da precipitação mensal, para o município de Cáceres (MT), de 1979 a 2009.

tendência, em estudos da bacia hidrográfica Paraguai/ Jauquara, localizada no Estado de Mato Grosso.

As menores médias mensais ocorreram nos meses de junho, julho e agosto, com 16,59 mm, $17,90 \mathrm{~mm}$ e $20,09 \mathrm{~mm}$, respectivamente, caracterizando o período mais crítico, em relação ao regime pluviométrico da região. Estes resultados corroboram os observados por Vela et al. (2007), que evidenciaram os meses de junho, julho e agosto como os de menores médias de precipitação pluviométrica, com $1,15 \mathrm{~mm}, 8,8 \mathrm{~mm}$ e $32,35 \mathrm{~mm}$, respectivamente, para a região de Tangará da Serra (MT). Já para o município de Nova Maringá, localizado ao norte de Mato Grosso, Moreira et al. (2010) constataram que o comportamento mensal da precipitação foi diferente dos evidenciados para Cáceres, pois os menores índices de precipitação ocorreram entre os meses de março e julho, demonstrando que, ao norte do Estado, o período de estiagem ocorre mais cedo, em relação à região do Pantanal Matogrossense.

De dezembro a março, foram observadas as maiores médias de precipitação, com $205,5 \mathrm{~mm}$, $243,8 \mathrm{~mm}, 186,4 \mathrm{~mm}$ e $177,3 \mathrm{~mm}$, respectivamente, com dezembro e janeiro apresentando médias superiores a $200 \mathrm{~mm}$. Dallacort et al. (2010) encontraram resultados similares para Diamantino (MT), pois, nesta localidade, os três meses mais chuvosos também foram dezembro, janeiro e fevereiro, com médias de $277,7 \mathrm{~mm}, 288,9 \mathrm{~mm}$ e $288,4 \mathrm{~mm}$, respectivamente. Além disto, os autores observaram que alguns municípios do Estado de Mato Grosso, como Diamantino, Tangará da Serra, Cuiabá, Campo Novo do Parecis e Cáceres, apresentaram características semelhantes, em relação ao regime pluviométrico.

A maior média de precipitação ocorreu no mês de janeiro $(243,58 \mathrm{~mm})$ e a menor em junho 
$(16,59 \mathrm{~mm})$. No período de seca, em nenhum dos meses foram verificadas médias superiores a $100 \mathrm{~mm}$.

Avaliando-se os desvios-padrão, observou-se que o mês de dezembro apresentou maior uniformidade de precipitação, com desvio-padrão de 72,54 mm. Por outro lado, o mês de janeiro apresentou maior desvio-padrão, com 89,88 mm.

A média de dias secos e chuvosos, da série de 26 anos, foi de 305 e 60 dias, respectivamente. Nos anos 1986 e 2002, ocorreram 320 dias secos, sendo estes os anos com maiores frequências de dias secos (Figura 3). Já 1992 foi o ano mais chuvoso, pois, neste, ocorreram 104 dias considerados chuvosos.

Para a região de Nova Maringá (MT), Moreira et al. (2010) constataram que 1999 foi o ano mais seco, com 317 dias sem chuva, e 1990 o de maior número de dias chuvosos. Já em Tangará da Serra (MT), Dallacort et al. (2009) verificaram que o ano mais crítico, em relação à seca, foi 1976, com apenas 75 dias chuvosos, sendo 1984 o ano mais chuvoso.

Na Figura 4, são apresentadas as ocorrências médias mensais de dias secos e chuvosos, sendo possível observar que, em todos os meses, a média de dias secos foi superior à de dias chuvosos. Tam-

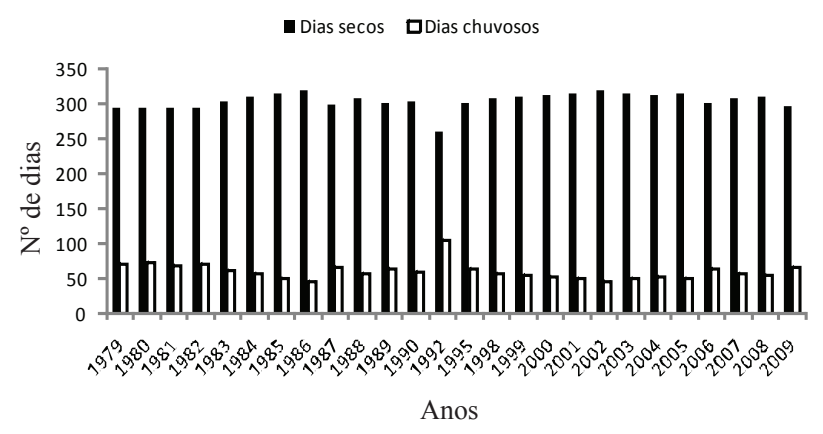

Figura 3. Ocorrência anual de dias secos e chuvosos, no município de Cáceres (MT), de 1979 a 2009.

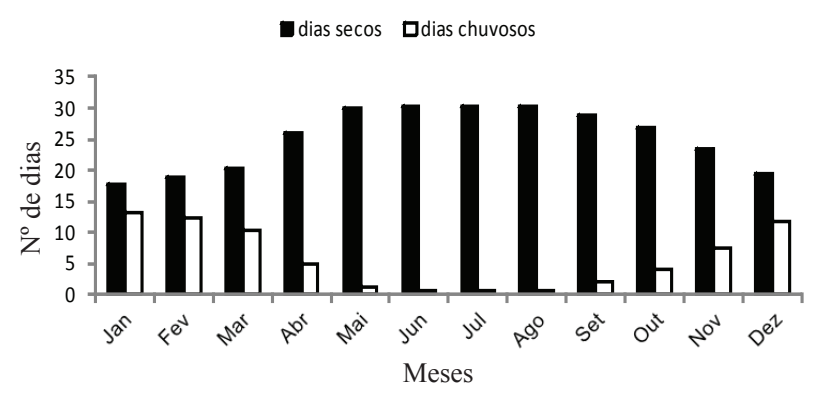

Figura 4. Ocorrência mensal média de dias secos e chuvosos, no município de Cáceres (MT), de 1979 a 2009. bém é possível constatar que, de julho a agosto, as precipitações são mais escassas, já que, neste período, a média de dias chuvosos ficou abaixo de um, ou seja, praticamente todos os dias foram considerados como secos.

Entre os meses de dezembro e março, as médias de dias chuvosos foram mais elevadas (superiores a 10 dias chuvosos). O mês de janeiro foi considerado o mais chuvoso, pois o mesmo apresentou média de 13 dias chuvosos (Figura 4). Para a sub-região de Nhecolândia, Pantanal Sul-Matogrossense, Soriano \& Galdino (2005) verificaram que janeiro também foi o mês com maior percentagem de dias chuvosos e que a frequência de precipitação mensal não é bem distribuída para a região.

Ao analisar as médias de precipitação dos meses mais chuvosos (dezembro e janeiro), observou-se que o mês de dezembro apresentou $40 \%$ de probabilidade de ocorrência de precipitações superiores a $200 \mathrm{~mm}$, enquanto janeiro apresentou $60 \%$ de probabilidade desta ocorrência. Com $90 \%$ de probabilidade de ocorrência, o período de dezembro a março apresentou probabilidade de precipitação superior a $100 \mathrm{~mm}$. Já o período de maio a agosto apresentou probabilidade de precipitação inferior a $6 \mathrm{~mm}$.

Os valores do parâmetro $\beta$ tiveram variações de 17,1 a 33,1 , não excedendo a 100 , em nenhum dos meses (Tabela 1), possibilitando a utilização da distribuição Gama, para as estimativas das precipitações prováveis, uma vez que valores de $\beta$ superiores a 100 indicam que a distribuição Gama incompleta não pode ser utilizada (Thom 1958, Araújo et al. 2001). O maior valor do parâmetro $\beta$ foi encontrado em janeiro, mês que apresentou precipitações mais intensas e maior desvio-padrão, com $243,8 \mathrm{~mm}$ e $89,9 \mathrm{~mm}$, respectivamente, indicando maior variabilidade de precipitação.

Constatou-se que o parâmetro $\alpha$ não excedeu a 100 em nenhum dos períodos estudados, com valores variando de 0,9 , em junho e agosto, a 13,4, em março. Os valores mais baixos coincidiram com os meses de menor volume de precipitação.

O modelo probabilístico de distribuição Gama incompleta (Thom 1958) é amplamente utilizado para a determinação da precipitação provável. Existem trabalhos nos quais o modelo apresentou ajuste adequado, dentre estes, alguns realizados no Estado de Mato Grosso (Fietz et al. 2008, Martins et al. 2010, Moreira et al. 2010). 
Tabela 1. Valores de $\alpha, \beta$ e precipitação provável, para o município de Cáceres (MT).

\begin{tabular}{|c|c|c|c|c|c|c|c|c|c|c|c|}
\hline \multirow{2}{*}{ Mês } & \multirow{2}{*}{$\bar{X}$} & \multirow{2}{*}{$\mathrm{S}^{2}$} & \multirow{2}{*}{$\alpha$} & \multirow{2}{*}{$\beta$} & \multicolumn{7}{|c|}{ Níveis de probabilidade } \\
\hline & & & & & $90 \%$ & $75 \%$ & $60 \%$ & $50 \%$ & $40 \%$ & $25 \%$ & $10 \%$ \\
\hline Jan & 243,8 & 89,9 & 7,4 & 33,1 & 138,0 & 178,7 & 211,4 & 232,8 & 255,7 & 296,9 & 363,7 \\
\hline Fev & 186,4 & 70,3 & 7,0 & 26,6 & 103,8 & 135,4 & 160,8 & 177,6 & 195,5 & 227,8 & 280,3 \\
\hline Mar & 177,3 & 48,4 & 13,4 & 13,2 & 118,7 & 142,7 & 161,1 & 172,9 & 185,2 & 207,1 & 241,4 \\
\hline Abr & 90,1 & 39,3 & 5,3 & 17,1 & 44,8 & 61,4 & 75,2 & 84,4 & 94,4 & 112,6 & 142,6 \\
\hline Mai & 35,0 & 31,8 & 1,2 & 28,8 & 5,1 & 12,1 & 19,9 & 26,0 & 33,3 & 48,4 & 76,9 \\
\hline Jun & 16,6 & 17,5 & 0,9 & 18,5 & 1,4 & 4,2 & 7,9 & 11,0 & 14,8 & 23,0 & 39,2 \\
\hline Jul & 17,9 & 17,7 & 1,0 & 17,6 & 2,0 & 5,2 & 9,2 & 12,5 & 16,5 & 24,8 & 41,0 \\
\hline Ago & 20,1 & 21,5 & 0,9 & 22,9 & 1,6 & 5,0 & 9,4 & 13,2 & 17,8 & 27,8 & 47,8 \\
\hline Set & 47,5 & 35,5 & 1,8 & 26,5 & 11,3 & 21,5 & 31,6 & 39,0 & 47,6 & 64,4 & 94,8 \\
\hline Out & 91,6 & 45,0 & 4,2 & 22,1 & 40,7 & 58,7 & 74,0 & 84,3 & 95,7 & 116,6 & 151,8 \\
\hline Nov & 133,4 & 62,1 & 4,6 & 29,0 & 62,5 & 88,0 & 109,4 & 123,9 & 139,6 & 168,5 & 216,6 \\
\hline Dez & 205,5 & 73,9 & 7,7 & 26,6 & 118,3 & 152,1 & 179,0 & 196,7 & 215,6 & 249,5 & 304,1 \\
\hline
\end{tabular}

\section{CONCLUSÕES}

1. A distribuição de chuvas na região de Cáceres (MT) apresentou grande variabilidade, no decorrer dos anos, variando de $972,9 \mathrm{~mm}$, em 1985, a 1.624,1 mm, em 1998.

2. A região apresenta duas estações bem definidas, sendo elas o período de seca (maio a setembro) e o chuvoso (outubro a abril).

3. A maior média de precipitação ocorreu no mês de janeiro, com 243,58 mm, e a menor em junho, com $16,59 \mathrm{~mm}$.

4. Dezembro, janeiro, fevereiro e março apresentam $90 \%$ de probabilidade de ocorrência de pluviosidade superior a $100 \mathrm{~mm}$.

\section{REFERÊNCIAS}

ARAÚJO, W. F. et al. Precipitação pluviométrica mensal provável em Boa Vista, Estado de Roraima, Brasil. Revista Brasileira de Engenharia Agrícola e Ambiental, Campina Grande, v. 5, n. 3, p. 563-567, 2001.

ASSIS, F. N.; ARRUDA, H. V.; PEREIRA, A. R. Aplicações de estatística à climatologia: teoria e prática. Pelotas: UFPel, 1996.

CASARIN, R.; SILVA NEVES, S. M. A.; NEVES, R. J. Uso da terra e qualidade da água da bacia hidrográfica Paraguai/Jauquara. Revista Geográfica Acadêmica, Cáceres, v. 2, n. 1, p. 33-42, 2008.

CUNHA, C. N. da; JUNK, W. J. Year-to-year changes in water level drive the invasion of Vochysia divergens in Pantanal grassland. Applied Vegetation Science, Uppsala, v. 7, n. 1, p. 103-110, 2004.
DALLACORT, R. et al. Aptidão agroclimática do pinhãomanso na região de Tangará da Serra, MT. Ciência Agronômica, Fortaleza, v. 41, n. 3, p. 373-379, 2010.

DALLACORT, R. et al. Frequência de dias secos e chuvosos na região de Tangará da Serra, MT. In: CONGRESSO BRASILEIRO DE ENGENHARIA AGRÍCOLA, 38., 2009, Juazeiro. Anais... Juazeiro: SBEA, 2009. 1 CD-ROM.

DINIZ, G. L.; FONSECA, M. Análise harmônica do regime de precipitação em duas localidades da baixada cuiabana. Biomatemática, Campinas, v. 18, n. 1, p. 3748, 2008.

FARIA, R. T. et al. CLIMA: programa computacional para organização e análise de dados meteorológicos. Engenharia Agricola, Jaboticabal, v. 23, n. 2, p. 372-387, 2003.

FIETZ, C. R. et al. Estimativa da precipitação provável para o Estado de Mato Grosso. Dourados: Embrapa Agropecuária Oeste, 2008.

IVERSSON, L. B. et al. Circulation of eastern equine encephalitis, western equine encephalitis, Ilhéus, Maguari and Tacaiuma viruses in equines of the Brazilian Pantanal, South America. Revista do Instituto de Medicina Tropical, São Paulo, v. 35, n. 4, p. 355-359, 1993.

MARTINS, J. A. et al. Probabilidade de precipitação para a microrregião de Tangará da Serra, Estado do Mato Grosso. Pesquisa Agropecuária Tropical, Goiânia, v. 40, n. 3, p. 291-296, 2010.

MOREIRA, P. S. P. et al. Distribuição e probabilidade de ocorrência das chuvas no município de Nova Maringá-MT. Revista de Ciências Agroambientais, Alta Floresta, v. 8, n. 1, p. 9-20, 2010. 
ROSA, D. B. et al. A distribuição espacial das chuvas na porção centro-oeste do Estado de Mato Grosso Brasil. Revista Eletrônica da Associação dos Geógrafos Brasileiros, Três Lagoas, v. 1, n. 5, p. 127-152, 2007.

SALGUEIRO, J. H. P. B. Avaliação de rede pluviométrica e análise de variabilidade espacial da precipitação: estudo de caso na bacia do Rio Ipojuca em Pernambuco. 2005. 139 f. Dissertação (Mestrado em Engenharia Civil)Universidade Federal de Pernambuco, Recife, 2005.

SANS, L. M. A. et al. Zoneamento de riscos climáticos para a cultura do milho na Região Centro-Oeste do Brasil e para o Estado de Minas Gerais. Revista Brasileira de Agrometeorologia, Santa Maria, v. 9, n. 3, p. 527-539, 2001.

SILVA, J. C. et al. Análise de distribuição de chuva para Santa Maria, RS. Revista Brasileira de Engenharia Agrícola e Ambiental, Campina Grande, v. 11, n. 1, p. 6772, 2007.
SORIANO, B. M. A.; GALDINO, S. Análise da distribuição da frequência mensal da precipitação para a sub-região da Nhecolândia, Mato Grosso do Sul, Pantanal, Brasil. Corumbá: Embrapa Pantanal, 2005. (Boletim de pesquisa, 34).

TENUTA, J. A. Pantanal: um pedaço do Brasil. Goiânia: R\&F, 2004.

THOM, H. C. S. A note on the Gama distribution. Monthly Weather Review, Washington, DC, v. 86, n. 4, p. 117-122, 1958.

VELA, R. H. N.; DALLACORT, R.; NIED, A. H. Distribuição decendial, mensal e totais de precipitação na região de Tangará da Serra - MT. In: CONGRESSO BRASILEIRO DE ENGENHARIA AGRÍCOLA, 36., 2007, Bonito. Anais... Bonito: SBEA, 2007. p. 1-4. 